tion can lead to fission of the rapidly collapsing core before collapse of the envelope has reached appreciable velocity. During the development of the prolate deformation which leads to fission, the core releases nuclear energy in amount $\sim 10^{-3} M c^{2} \sim 10^{59}$ ergs into the envelope. This energy is sufficient to meet the luminosity requirement of the radio stars for $10^{5}$ to $10^{6}$ years. Upon fission the binary components collapse in $\sim 0.1$ year to their gravitational radii. A turbulent, quasi-stable envelope of convecting, radiating material surrounds the rotating binary system. Other more complicated nonspherical internal structures could conceivably support the radiating envelope.

(3) Appropriate choices for the parameters involved can be made which lead to lifetimes for the binary system also in the range $10^{5}$ to $10^{6}$ years. In a relatively short interval ( $\sim 0.1$ year) at the end of this period, gravitational radiation from the rotating binary, which does have a quadrupole moment, injects energy into the envelope material in amount $\sim 10^{-2} M c^{2} \sim 10^{60}$ ergs. It is suggested that the resulting polar explosion may lead to the development of the strong, extended radio sources with at least two components.

(4) On the model discussed it is found that the gravitational resources of a massive star exceed the nuclear resources by only a factor of ten. Only $1 \%$ of the rest mass energy is made available for all forms of radiation. This and other problems are noted briefly at the end of Part III.

\section{ACKNOWLEDGMENTS}

The author is indebted to many of his colleagues in physics and astronomy at the California Institute of Technology and the Mt. Wilson-Palomar Observatories for discussions of the subject matter of this paper. He is especially indebted to Professor Murray Gell-Mann who reopened the question of gravitational radiation, in particular that from a rotating binary system, and who collaborated in estimating the importance of gravitational radiation in collapsing systems. To Fred Hoyle, Geoffrey Burbidge, and Margaret Burbidge, he is indebted for constant collaboration over the past year on the subject of massive stars.

\title{
The Calculation of Stellar Pulsation*
}

\author{
ROBERT F. CHRISTY \\ California Institute of Technology, Pasadena, California
}

\section{INTRODUCTION}

In this paper we report methods of computation which have been developed to provide a theoretical understanding of the RR Lyrae and Cepheid type pulsating stars. The results reported are intended to illuminate the methods of calculation and to provide insight into the physical processes in these stars. A survey of pulsation in RR Lyrae models ${ }^{1,2}$ using these methods has also been carried out and will be reported soon in another journal. A survey of pulsation in Cepheid models has been initiated and will be continued. $^{3}$

\footnotetext{
* Work supported in part by the Office of Naval Research and the National Aeronautics and Space Administration.

${ }^{1}$ R. F. Christy, Astron. J. 68, 275 (1963).

2 R. F. Christy, Astron. J. 68, 534 (1963)

3 A. N. Cox, K. H. Olsen, and J. P. Cox [Astron. J. 68, 276 (1963)] have reported some somewhat similar calculations on Cepheid models. Unfortunately, they have not included the deeper regions of the envelope or the hydrogen ionization region near the surface. As a result, their calculations cannot be compared in detail with the observations.
}

The methods reported here arose from investigations $^{4}$ (referred to as I) on the energy transport in the hydrogen ionization zone of giant stars. In that paper, some preliminary numerical integrations of the equations of motion were reported, and the possibility of spontaneous generation of oscillations or pulsation was demonstrated. The machine code used at that time was, however, not suitable for more extensive calculations and the work reported on here is the refinement and extension of the earlier calculations.

The general idea behind these calculations is that the observed pulsation motions in Cepheids and RR Lyrae (and other related) stars arise spontaneously because of the particular physical properties of the envelopes. The relevant physical properties are the equation of state and the opacity. The method of attack is to integrate the time-dependent equations of hydrodynamics (with spherical symmetry) and heat flow by numerical means.

\footnotetext{
${ }^{4}$ R. F. Christy, Astrophys. J. 136, 887 (1962).
} 
Small amplitude linearized calculations on this problem have been carried out recently by a number of authors. ${ }^{5-7}$ These calculations left many questions, particularly about the role of hydrogen ionization, unanswered. These calculations were also unable to achieve results that agreed with many characteristic features of the observations-such as the phase lag of the luminosity curve. It was to answer these questions and to be able to compare the results directly with the large amplitude observed pulsations that the present program was pursued.

\section{EQUATIONS OF MOTION}

The mass interior to $r$ is given by

$$
M(r)=\int_{0}^{r} 4 \pi r^{\prime 2} \rho\left(r^{\prime}\right) d r^{\prime},
$$

where $\rho(r)$ is the density at $r$. Then the specific volume

$$
V=1 / \rho(r)=4 \pi r^{2} d r / d M .
$$

We use Lagrangian coordinates attached to the mass element $r$ so that $r(M, t)$ is the position of a given mass element. Then Newton's equation is

$$
\frac{\partial^{2} r}{\partial t^{2}}=-\frac{G M}{r^{2}}-\frac{1}{\rho} \frac{\partial P}{\partial r},
$$

or

$$
\frac{\partial^{2} r}{\partial t^{2}}=-\frac{G M}{r^{2}}-4 \pi r^{2} \frac{\partial P}{\partial M},
$$

where $P$ is the pressure at $M$.

The heat flow is assumed to be entirely through radiation diffusion and the total luminous flux at some level is given by

$$
L(r)=-4 \pi r^{2} \frac{4 \sigma}{3} \frac{d\left(T^{4}\right)}{\kappa(V, T) \rho(r) d r},
$$

where $\sigma$ is the Stefan-Boltzmann constant and $\kappa(V, T)$ is the (Rosseland mean) opacity in $\mathrm{cm}^{2} / \mathrm{g}$. We can write

$$
L=-\left(4 \pi r^{2}\right)^{2} \frac{4 \sigma}{3 \kappa} \frac{d\left(T^{4}\right)}{d M}
$$

using $M$ as the independent variable. Then the heat flow equation becomes

$$
T \frac{\partial S}{\partial t}=\frac{\partial Q}{\partial t}=-\frac{d}{d M}(L)
$$

\footnotetext{
${ }^{5}$ S. A. Zhevakin, Astron. Zh. 30, 161 (1953).

${ }^{6}$ N. Baker and R. Kippenhahn, Z. Astrophys. 54, 114 $(1962)$

${ }^{7}$ J. P. Cox, Astrophys. J. 138, 487 (1963).
}

where $S$ is the entropy/g and $Q$ the heat conducted/g. Writing

$$
T d S=d E+P d V
$$

where $E$ is the internal energy/g, we have

$$
\frac{\partial E}{\partial t}+P \frac{\partial V}{\partial t}+\frac{d L}{d M}=0
$$

Energy conservation is vital for a discussion of dynamics. We can form a mechanical energy equation by multiplying Newton's equation by $\dot{r}$, so

$$
\begin{aligned}
\frac{d}{d t}\left(\frac{1}{2} \dot{r}^{2}-\frac{G M}{r}\right) & =-4 \pi r^{2} \dot{r} \frac{\partial P}{\partial M} \\
& =-\frac{d}{d M}\left(4 \pi r^{2} \dot{r} P\right)+P \frac{d V}{d t} .
\end{aligned}
$$

The left-hand side represents the rate of change of the kinetic plus gravitational energy per gram. The first term on the right is the divergence of the flux of mechanical energy and the second term represents the work done.

This equation can be combined with the heat equation to give the over-all energy conservation equation

$\frac{d}{d t}\left(\frac{1}{2} \dot{r}^{2}-\frac{G M}{r}+E\right)+\frac{d}{d M}\left(4 \pi r^{2} \dot{r} P+L\right)=0$.

The above theory is incomplete in that it omits convection. It is hoped to include a dynamical form of convection theory at a later date.

\section{EQUATIONS OF STATE AND OPACITY}

The equation of state was obtained by assuming a perfect gas of $\mathrm{H}, \mathrm{H}^{+}, \mathrm{He}, \mathrm{He}^{+}, \mathrm{He}^{++}$, and electrons. $\mathrm{H}_{2}$ (molecules) were ignored as being unimportant in consideration of the RR Lyrae and Cepheid instability strip. The relative numbers of the various ions were determined by solving the Saha equations of equilibrium, ignoring pressure ionization, ionic interaction, etc. This is a very good approximation for the very low density envelopes of these particular stars. The contribution of the various ionic species to the pressure and internal energy (including the ionization energy) was computed.

The opacities used were Rosseland mean opacities obtained from A. N. Cox and J. N. Stewart (unpublished) of Los Alamos Scientific Laboratory in summer 1962. We are very grateful for the use of these results prior to publication. These calculations included the effects of bound-bound transitions in addition to the usual bound-free, free-free, and scattering contributions. The calculations were aug- 
mented by additional calculations ${ }^{8}$ of the continuum opacity, excluding bound-bound transitions, in the photospheric region. Interpolation formulas were developed to provide a more convenient and continuous representation of the computed opacities. Details of these expressions will be published in another paper dealing with a survey of RR Lyrae stars.

In I (p. 889) it was pointed out that there was a maximum flux that could be transported by convection $H_{c} \approx 10^{6} P \mathrm{ergs} / \mathrm{cm}^{2}$ sec, where $P$ is the pressure at $\sim 10^{4}{ }^{\circ} \mathrm{K}$ corresponding to the extreme of transport of ionized hydrogen at a velocity near sonic. Also an estimate of $P$ at $10^{4}{ }^{\circ} \mathrm{K}$ (the ionization temperature) gave $P^{\frac{3}{2}} \approx 7\left[g_{e} /\left(T_{e} / 10^{4}{ }^{\circ}\right)^{13}\right]$. These estimates, if translated to a line on the HertsprungRussel diagram by use of a mass-luminosity relation, correspond to a line just to the right (or slightly lower $T_{e}$ ) than the line marking the Cepheid variables and RR Lyrae stars. In fact, the limiting convective flux is about $10 \%$ of the total flux of such variables. We here use this result to eliminate further consideration of convection as a means of heat transport for these stars. This omission is probably not serious in the center or on the high temperature side of the unstable region. However, a suggestion arises in this work that it is the onset of effective convection that determines the low temperature boundary of instability in the Cepheid and RR Lyrae stars that we will investigate.

\section{THE BOUNDARY CONDITIONS}

The calculation of the motion of the envelope will be carried out omitting the core of the star where nuclear energy is made available. This requires boundary conditions on the mechanics and on the heat flow both at the inner and at the outer boundary. It is essential that these boundary conditions be formulated in such a manner as to not falsify the damping or undamping of the oscillations.

Rabinowitz ${ }^{9}$ showed for stars with giant envelopes that the amplitude of the motion is exceedingly small at small radii. For this reason, he found that the coupling with nuclear energy generation in the core was negligible. This suggests treating the central core as an inert heat source. Ultimately this boundary condition can be tested by varying the position where it is applied and checking the lack of sensitivity of the result. The physical properties we assign to the core are a constant rate of generation of heat energy and no generation of mechanical energy. It

${ }^{8} \mathrm{I}$ want to thank J. Noble for assistance in performing the calculations.

${ }^{9}$ I. N. Rabinowitz, Astrophys. J. 126, 386 (1957). might be possible to represent these properties in an adiabatic gas sphere but the complications involved in a finite velocity of sound have led us to use a rigid sphere at the center. This means that at $r=R_{1}$, $U=\dot{r}=0$. The constant heat source is given by $L\left(R_{1}\right)=L_{0}$. It is clear that the condition $U=0$ means that no mechanical energy is transmitted across the boundary.

At the upper boundary there are also complications. It is straightforward to propose the appropriate treatment of the heat flow: it should be done by a time-dependent and wavelength-dependent integral equation of transfer. This problem has been examined and it would greatly complicate the subsequent calculation. The purpose of the present calculation is to follow the general features of the motion sufficiently accurately to compare with observation, but it is not intended that the calculation should give all the details necessary for the understanding of spectra.

The static, gray atmosphere, solution of the equation of transfer is

$$
T^{4}=\frac{3}{4} T_{e}^{4}[\tau+q(\tau)],
$$

where $\tau$ is the optical depth and $q(\tau)$ is to be found in Chandrasekhar. ${ }^{10} \mathrm{~A}$ fairly close approximation to this expression can be obtained as a solution of the time-independent diffusion equation. It is

$$
T^{4}=\frac{3}{4} T_{e}^{4}(\tau+c),
$$

where $c$ is a constant. We have, therefore, chosen a boundary condition on the time-dependent diffusion equation which is consistent with this form for the time-independent problem. We have taken $c=\frac{2}{3}$. This condition is written as

$$
d\left(T^{4}\right) /\left.d \tau\right|_{\text {surface }}=\frac{3}{4} T_{e}^{4}=T^{4} /\left.\frac{2}{3}\right|_{\text {surface }} .
$$

If we calculate to the strict outer boundary of the star (where the density vanishes), then the mechanical energy flux must vanish since $P=0$. Strictly speaking, we are not entirely sure where this boundary is since the star may be evaporating or losing mass by more violent means. We have chosen to define the boundary of the star by $P=0$ which defines the outer boundary condition. An accurate dynamical calculation might show that this boundary steadily expanded and left the body of the star. This would give rise to a damping of the mechanical energy by the continual ejection of material. This kind of result is not precluded by the condition $P=0$, however, the accurate treatment of such a motion (involving mass ejection), would require the consid-

${ }^{10}$ S. Chandrasekhar, Radiative Transfer (Dover Publications, Inc., 1960). 
eration of the motion of very thin layers. Such layers would be optically thin and all of the basic physics involved would have to be reviewed and adjusted to these conditions. For these reasons, we will not attempt the calculation of mass loss in this paper. We can, however, consider sufficiently thin layers in the atmosphere that the effects of the propagation of acoustic waves, the formation of shock waves, and consequent dissipation of mechanical energy are taken into account.

\section{INITIAL CONDITIONS}

The procedure to be followed is the solution of the time-dependent partial differential Eqs. (2) and (4) subject to the boundary conditions outlined above. This is to be a time-dependent solution and will depend on the initial conditions.

We could start from arbitrary initial conditions for $T(M), V(M)$, and velocity $U(M)$ : presumably in the course of time, the solution would settle down to the correct final state, either static or pulsating as the case might be. However, it is clear that this would take a long time since the initial thermal energy content, being incorrect, would have to relax in the time required for thermal relaxation of the inner envelope which is very long compared to the pulsation period.

Since it is known that the amplitudes of pulsation are small in the deep interior, we suppose that the solution for $T(M)$ and $V(M)$ do not differ greatly from the static solution in the inner envelope. We therefore base our initial conditions for the timedependent problem on the solution of the static envelope problem for the same star. It would be possible to use this static solution as the initial condition: if the envelope was unstable, small errors in the static solution would initiate a pulsation which would grow until the amplitude asymptotically leveled off at some final value. This is not, in most cases, a practical procedure because of the excessively long computation involved.

We have chosen, instead, to initiate the pulsation by suitably modifying the static solution and following the time-dependent behavior. The best procedure would involve choosing initial values for $T(M)$, $V(M), U(M)$ that would be consistent with a natural periodic motion of the star. This is not possible since we don't know this motion. Actually, there are many (infinitely) modes of motion of the envelope and we wish to consider only the lowest ones, and only one at a time. The best way we have found for doing this is to start with the static solutions for $T(M)$ and $V(M)$ and superimpose some arbitrary $U(M)$. This at least has the advantage that the pressure is initially in equilibrium so that we do not initiate arbitrary sound waves and shock waves. A disadvantage of this procedure is that the true solution for a single harmonic never passes through this condition (because of nonlinearities and phase shifts), especially in the nonadiabatic region. This procedure necessarily then initiates a mixture of harmonics. Of this mixture, only the few lowest harmonics survive very long.

\section{THE DIFFERENCE EQUATIONS}

In order to integrate numerically the partial differential equations (2) and (4) of motion, it is necessary to express them as difference equations. Although there are many ways of doing this, they are restricted by the requirements of stability and accuracy. Up to a point, these questions are subject to analytic examination but in highly nonlinear problems and in problems involving the coupling of hydrodynamics and heat conduction, as this does, the approach is in large part based on experience. Richtmyer $^{11}$ discusses these problems individually but not coupled. By far the principal experience with the coupled problem of this kind is to be found at the Atomic Energy Commission Laboratories at Los Alamos initially, and also at Livermore: unfortunately, most of this experience has not been made available. The procedure used here was developed after reading Richtmyer. A Los Alamos report ${ }^{12}$ was helpful on hydrodynamic questions, and a paper by Henyey et al. ${ }^{13}$ was helpful on the treatment of heat flow. Although the method developed here seems satisfactory, it cannot be claimed that it represents the highest state of the art (and it still is an art), which remains in laboratories like those mentioned above.

The variable $R(M, t)$ is represented by a discrete quantity $R_{n}(I)$ where the index $n$ (integer) represents the time $t^{n}$ and the index $I$ (integral) represents the mass $M(I)$ internal to $R^{n}(I) . I=1$ will represent the inner boundary and $I=N$ the outer. The mass between $I$ and $I-1$ is given by

$$
\Delta M\left(I-\frac{1}{2}\right)=M(I)-M(I-1) .
$$

The specific volume of the mass element at $I-\frac{1}{2}$ is

$$
\begin{aligned}
& V^{n}\left(I-\frac{1}{2}\right) \\
& \quad=\frac{4}{3} \pi\left\{\left[R^{n}(I)\right]^{3}-\left[R^{n}(I-1)\right]^{3}\right\} / \Delta M\left(I-\frac{1}{2}\right) .
\end{aligned}
$$

${ }_{11}$ R. D. Richtmyer, Difference Methods for Initial Value Problems (Interscience Publishers, Inc., New York, 1957).

12 J. E. Fromm, Lagrangian Difference Approximations for Fluid Dynamics (Office of Technical Services, Department of Commerce, Washington, D. C., 1961), LA-2535.

${ }^{13}$ L. G. Henyey, L. Wilets, K. H. Böhm, R. Le Levier, and R. D. Levee, Astrophys. J. 129, 628 (1959). 
$T^{n}\left(I-\frac{1}{2}\right)$ and $P^{n}\left(I-\frac{1}{2}\right)$ are the mean temperature and pressure (at time $t$ ) of the mass element at $I-\frac{1}{2}$.

For best centering in time, the velocity is defined at time $t^{n}+\frac{1}{2} \Delta t$, i.e., it is labeled in time by $n+\frac{1}{2}$. It is $U^{n+\frac{1}{2}}(I)$. Then we have

$$
R^{n+1}(I)=R^{n}(I)+\Delta t^{n+\frac{1}{2}} U^{n+\frac{1}{2}}(I)
$$

where $\Delta t^{n+\frac{1}{2}}$ is the time between $t^{n}$ and $t^{n+1}$. This is kept separate from $\Delta t^{n}$ which is the time between $t^{n-\frac{1}{2}}$ and $t^{n+\frac{1}{2}}$ since $\Delta t$ will be changed from time to time in order to satisfy the stability conditions. The time scale is defined by $t^{n+1}=t^{n}+\Delta t^{n+\frac{1}{2}}$ and $t^{n+\frac{1}{2}}=$ $t^{n-\frac{1}{2}}+\Delta t^{n}$, where $\Delta t^{n}=\frac{1}{2}\left(\Delta t^{n-\frac{1}{2}}+\Delta t^{n+\frac{1}{2}}\right)$.

Shock waves are treated by the Von NeumannRichtmyer method (Ref. 11, p. 216), which involves the introduction of an artificial "viscosity" which creates a pressure on rapid compression but none on expansion. The viscous pressure is given by

$$
\begin{aligned}
Q^{n-\frac{1}{2}}\left(I-\frac{1}{2}\right) & =C_{Q} \frac{\left[U^{n-\frac{1}{2}}(I)-U^{n-\frac{1}{2}}(I-1)\right]^{2}}{V^{n}\left(I-\frac{1}{2}\right)+V^{n-1}\left(I-\frac{1}{2}\right)} \\
& =0 \quad \text { if } U(I)-U(I-1)<0,
\end{aligned}
$$

In this expression, the constant $C_{Q}$ is chosen as a compromise between the requirements of stability and accuracy. The effect of the pressure $Q$ is to artificially spread the shock front over several mass points. A larger value of $C_{Q}$ makes a thicker shock front but greater stability.

The equation of motion is then, for $I=2$ to $N-1$,

$$
\begin{aligned}
U^{n+\frac{1}{2}}(I)= & U^{n-\frac{1}{2}}(I)-\Delta t^{n}\left\{\frac{G M(I)}{\left[R^{n}(I)\right]^{2}}\right. \\
& +\frac{4 \pi\left[R^{n}(I)\right]^{2}}{\Delta M(I)}\left[P^{n}\left(I+\frac{1}{2}\right)-P^{n}\left(I-\frac{1}{2}\right)\right. \\
& \left.\left.+Q^{n-\frac{1}{2}}\left(I+\frac{1}{2}\right)-Q^{n-\frac{1}{2}}\left(I-\frac{1}{2}\right)\right]\right\}
\end{aligned}
$$

where

$$
\Delta M(I)=\frac{1}{2}\left[\Delta M\left(I+\frac{1}{2}\right)+\Delta M\left(I-\frac{1}{2}\right)\right] .
$$

The boundary conditions are, at $I=1, U^{n+\frac{1}{2}}(1)=0$, and for $I=N$ we define a fictitious pressure at $N+\frac{1}{2}$ which is the negative of $P\left(N-\frac{1}{2}\right)$, thereby insuring $P=0$ at $R(N)$. Then

$$
\begin{aligned}
& U^{n+\frac{1}{2}}(N)=U^{n-\frac{1}{2}}(N)-\Delta t^{n}\left\{\frac{G M(N)}{\left[R^{n}(N)\right]^{2}}\right. \\
& \left.-\frac{4 \pi\left[R^{n}(N)\right]^{2}}{\Delta M\left(N-\frac{1}{2}\right)}\left[2 P^{n}\left(N-\frac{1}{2}\right)+Q^{n-\frac{1}{2}}\left(N-\frac{1}{2}\right)\right]\right\} .
\end{aligned}
$$

\section{HEAT CONDUCTION}

We have used $T^{4} \equiv W$ as the temperature variable since it is approximately linear in $M$ over much of the envelope so that the difference equations will be more exact. It is not known, however, whether this in fact resulted in any reduction of error. The total luminous flux through radius $R^{n}(I)$ is given by

$$
\begin{aligned}
L^{n}(I)=\left\{4 \pi\left[R^{n}(I)\right]^{2}\right\}^{2}\left[W^{n}\left(I-\frac{1}{2}\right)\right. & \\
& \left.-W^{n}\left(I+\frac{1}{2}\right)\right] 2 F^{n}(I)
\end{aligned}
$$

where $2 F^{n}(I)$ is a suitable difference approximation to $\frac{4}{3} \sigma / \kappa \Delta M$. A possible expression would be

$$
\begin{aligned}
F^{n}(I)=\frac{4}{3} \sigma /\left[\kappa^{n}\left(I+\frac{1}{2}\right) \Delta M\right. & \left(I+\frac{1}{2}\right) \\
& \left.+\kappa^{n}\left(I-\frac{1}{2}\right) \Delta M\left(I-\frac{1}{2}\right)\right]
\end{aligned}
$$

where $\kappa\left(I+\frac{1}{2}\right)$ is the opacity in $\mathrm{cm}^{2} / \mathrm{g}$ at $I+\frac{1}{2}$.

An appropriately centered energy transport equation is then

$$
\begin{aligned}
& \left(E^{n+1}\left(I+\frac{1}{2}\right)-E^{n}\left(I+\frac{1}{2}\right)+\left\{\frac { 1 } { 2 } \left[P^{n}\left(I+\frac{1}{2}\right)\right.\right.\right. \\
& \left.\left.+P^{n+1}\left(I+\frac{1}{2}\right)\right]+Q^{n+\frac{1}{2}}\left(I+\frac{1}{2}\right)\right\}\left[V^{n+1}\left(I+\frac{1}{2}\right)\right. \\
& \left.\left.-V^{n}\left(I+\frac{1}{2}\right)\right]\right) \Delta M\left(I+\frac{1}{2}\right)=\frac{1}{2} \Delta t^{n+\frac{1}{2}}\left[L^{n+1}(I)\right. \\
& \left.\quad+L^{n}(I)-L^{n+1}(I+1)-L^{n}(I+1)\right]
\end{aligned}
$$

which has been time centered at $n+\frac{1}{2}$ and space centered at $I+\frac{1}{2}$. ( $E$ is the internal energy.) This form of the energy equation, which involves the new (not yet computed) temperatures at three adjacent mass points, is an implicit (Ref. 11, p. 91 et seq.) form of the heat conduction equation. ${ }^{14}$ Experience with linear forms of the equation suggests that this should be unconditionally stable for arbitrary time intervals.

The solution of this equation for the new temperatures at $n+1$ presents an additional problem since the equation is nonlinear. One possible method would be to expand and linearize the equation and solve as a set of $N$ coupled linear equations. We have instead chosen to solve by a process of iteration. The iteration is continued until the solution converges to any desired accuracy. This process of iteration, to-gether with the boundary conditions, and difference approximations, sometimes fails to converge. Each

${ }^{14}$ The reason that an implicit form of the heat conduction equation is so necessary for stability as well as to make physical sense can be understood as follows. The explicit form of the equation computes the new temperature from knowledge of the old temperature at three adjacent points. Thus, temperature information is able to propagate at most one mass point per time cycle. In a region of low heat capacity or high conductivity (such as near the stellar surface), however, the heat is able to propagate in fact over what may be many mass points in one time cycle. The implicit form of the equation, which makes a simultaneous solution for all new temperatures, clearly permits temperature information to propagate all the way from the boundaries in each time cycle, and thereby is able to correspond to reality. 
time this situation has arisen, it has been possible to so modify the procedure as to avoid the difficulty.

In the solution of the $N$ coupled linear equations which arise in the course of iteration, attention is drawn to the rapid procedures outlined in Richtmyer (p. 101). Conventional matrix inversion would be quite unsuitable in this connection.

The boundary condition at $I=1$ is

$$
\begin{aligned}
L^{n+1}(1)=\left\{4 \pi\left[R^{n+1}(1)\right]^{2}\right\}^{2}[ & W^{n+1}\left(\frac{1}{2}\right) \\
& \left.-W^{n+1}\left(\frac{3}{2}\right)\right] 2 F^{n+1}(1)=L_{0},
\end{aligned}
$$

where $L_{0}$ is the mean luminosisy. This equation serves to determine $W^{n+1}\left(\frac{1}{2}\right)$. The boundary condition at $I=N-\frac{1}{2}$ is

$$
\begin{aligned}
& \left(E^{n+1}\left(N-\frac{1}{2}\right)-E^{n}\left(N-\frac{1}{2}\right)+\left\{\frac { 1 } { 2 } \left[P^{n}\left(N-\frac{1}{2}\right)\right.\right.\right. \\
& \left.\left.\quad+P^{n+1}\left(N-\frac{1}{2}\right)\right]+Q^{n+\frac{1}{2}}\left(N-\frac{1}{2}\right)\right\}\left[V^{n+1}\left(N-\frac{1}{2}\right)\right. \\
& \left.\left.\quad-V^{n}\left(N-\frac{1}{2}\right)\right]\right) \Delta M\left(N-\frac{1}{2}\right)=\frac{1}{2} \Delta t^{n+\frac{1}{2}}\left\{L^{n+1}(N-1)\right. \\
& \quad+L^{n}(N-1)-2 \sigma 4 \pi\left[R^{n}(N-1)\right]^{2} W^{n}\left(N-\frac{1}{2}\right) \\
& \left.\quad-2 \sigma 4 \pi\left[R^{n+1}(N-1)\right]^{2} W^{n+1}\left(N-\frac{1}{2}\right)\right\} .
\end{aligned}
$$

This expression incorporates the boundary condition that the luminosity is $2 \sigma 4 \pi R^{2} T_{s}^{4}$, where $T$, is the surface temperature. The radius $R(N-1)$ is chosen rather than $R(N)$ since it is subject to much less error and allows to some extent for curvature.

Throughout the setting up of the difference equations, there are many alternative approaches. To some extent, these have been explored by others and the present method has been guided by the Refs. 11, 12, and 13. However, to a large extent in this problem, the various alternate approaches have not been explored. It is not known whether better approaches exist. The point of view has been to continue adjusting and correcting the procedure until it seemed satisfactory.

\section{THE MASS DIVISION}

In order to explore in more detail the structure of the envelope, it is useful to examine a simple model -namely a plane parallel example. Let $H$ be the radiative flux $/ \mathrm{cm}^{2}$ sec; $P$ is the gas pressure (we neglect radiation pressure), $g$ is the acceleration of gravity (assumed to be constant), and $m$ is the mass $/ \mathrm{cm}^{2}$ above a certain layer. Then the static pressure distribution is given by $P=m g$ and

$$
\frac{4}{3}(\sigma / \kappa) d\left(T^{4}\right) / d m=H=\sigma T_{e}^{4} .
$$

Now we find that a good approximation to $\kappa$ at temperatures above $2 \times 10^{4}$ is $\kappa=a+b P\left(10^{4} / T\right)^{4}$. If we now consider the equation for the variable
$P / T^{4}$, we see that the solution rapidly tends to

$$
\frac{4}{3}\left(g / \kappa T_{e}^{4}\right)=P / T^{4} .
$$

If we use the expression for $\kappa$ above, we can readily solve for $P / T^{4}$ which tends to a constant, implying that $\kappa$ tends to a constant. We are interested particularly in cases where $\kappa \gg a$. There results

$$
\kappa=\left[\frac{4}{3} g b /\left(T_{e} / 10^{4}\right)^{4}\right]^{\frac{1}{2}} .
$$

For the case of $45 \% \mathrm{He}$ and $0.2 \%$ heavy elements that we will discuss later, these relations give

$$
\left(\frac{T}{10^{4}}\right)^{4} \approx 0.13 \frac{P}{g^{\frac{1}{2}}}\left(\frac{T_{e}}{10^{4}}\right)^{2}
$$

and

$$
\kappa=0.17 g^{\frac{1}{2}}\left(10^{4} / T_{e}\right)^{2} .
$$

Because of these relations, we have used the variable $W \equiv T^{4}$ since it is approximately linear in $P$ and $m$.

The Courant condition (Ref. 11, p. 218) requires

$$
l=c \Delta t / \Delta R \leqslant l_{0} \approx 1,
$$

where $c$ is the velocity of sound. If we approximate $c \approx(P / \rho)^{\frac{1}{2}}$, we have

$$
=\frac{c \Delta t}{\Delta R}=\frac{P \Delta t}{c \rho \Delta R}=\frac{m g \Delta t}{c \Delta m}
$$

Thus, if $\Delta m / m$ is kept constant, $l$ decreases slowly as the temperature increases. Actually, where convergence sets in toward the center, $l$ increases again. For these reasons, we have used approximately $\Delta m / m=$ const, or what is the same, the ratio of successive values of $\Delta M$ is constant.

Now the number of mass points is proportional to $m / \Delta m$ and the time to compute one time cycle is proportional to the number of mass points. Further, $\Delta t$, as restricted by the Courant condition, varies roughly inversely as the number of mass points and the number of time cycles per period of oscillation is proportional to the number of points. Therefore, we see that the time to compute one period is proportional to the square of the number of mass points. This means that the mass division and the Courant condition must be carefully watched and the minimum number of mass points must be used consistent with the accuracy required. It was found that 30 to 40 points was sufficient for a reasonably accurate survey and, under these conditions, one period of the motion took about 100 time cycles. Under these conditions, one time cycle took about $\frac{1}{2}$ sec on a 7090 computer, one period took about 1 minute and a typical run was 600 time cycles, or about 6-7 
minutes, and covered 6-7 periods of the motion. Occasionally, calculations with up to 100 mass points have been used but it was, in general, not practical to follow these for very many periods except for a very few special cases.

The requirements represented by the Courant condition represent one reason why the core of the star should not be calculated in detail unless it is the seat of essential elements of the physics. The velocity of sound in the core is very great and if it were divided into sufficient mass points to give some detail in its behavior, the most severe restriction on the time interval would arise in the core and the number of cycles to compute a period of oscillation would probably increase by an order of magnitude.

In order to cover the envelope in about 35 steps, since $m$ varies over $\sim 7$ powers of 10 , it is necessary to use $\alpha[=\Delta M(I-1) / \Delta M(I)]$ up to 1.5 . This large fractional change per division might seem too large for accuracy; however, checks will be shown later which demonstrate the validity of the results.

In the region around $10^{4}$, the opacity law changes abruptly from a strongly increasing function of temperature in the photosphere to a decreasing one in the region of higher temperatures discussed above. Associated with this abrupt change in opacity, we also have hydrogen changing its ionization state. The net result of these conditions is that a static integration shows the temperature increasing very abruptly with depth in this region (see Figs. 3 and 4). If the mass is divided according to the discussion above, we find a near discontinuity in temperature, internal energy, opacity, at this point. The variable $W=T^{4}$ jumps by a factor of $>10$ and, at most, one mass point lies in the region of partial hydrogen ionization. At just the same region (actually for $T$ just below $10^{4}{ }^{\circ}$ ), the addition of the electrons to hydrogen causes a very rapid increase of density by a factor approaching 2 as the temperature falls, providing hydrogen is abundant. This increase of density of the higher layers is, of course, also responsible for the violent convective instability of this region. This density increase also reduces $\Delta R$ suddenly so that if $\alpha$ is constant, the most severe limitation on $\Delta t$ from the Courant condition arises just in these same layers.

This now poses a quandary: on the one hand, the accurate treatment of radiative transport demands an especially small $\Delta M$ just in the hydrogen ionization zone. On the other hand, the requirements of rapid calculation and stability demand an extra large $\Delta M$ in this zone. We have chosen an approximately constant value of $\alpha$ in this region in order to make rapid calculations and have given special attention to the energy transport so that a satisfactory treatment could be found in spite of the discontinuity in physical properties associated with the relatively large $\Delta M$.

The method of treating the heat transport equation is discussed in the next section. It has the property that the relation of temperature change to heat flow across this region is not precise under most conditions but is accurate on the average. As a result, if the zone of discontinuity-which moves up and down during the pulsation-moves over several mass zones during one period, then the average behavior of the discontinuity zone is correct. During a period, the temperature oscillates as the discontinuity moves across a mass zone but if there are several oscillations to a period, they can be averaged out.

The condition that the discontinuity cross several mass zones during a period prevents these calculations giving accurate results on the luminosity curve except for fairly large amplitude motions. Thus, it is not easy to extend the calculations accurately into the very small amplitude range where the linear theory should be correct.

This condition that the region near $10^{4}{ }^{\circ}$ should cross several mass zones is also related to the efficiency of convection. Thus the number of cycles to ionize one mass point is $q=\epsilon \Delta m / H \Delta t$, where $\epsilon$ is the ionization energy/g and we require $q$ not too large. Also, $l=c \Delta t / \Delta R \leqslant \rho c \Delta t / \Delta m$. A ratio that is relevant in the efficiency of convection is the ratio of the velocity of ionized matter required to transport the heat flux to the velocity of sound. This ratio $\beta$ should be small if convection is to be able to transport the heat. We have

$$
\beta=\frac{H}{\rho \epsilon c}=\frac{H \Delta t}{\Delta m} \frac{\Delta m}{\rho c \Delta t}=\frac{1}{q l} .
$$

Therefore, for $l$ fixed $\leqslant l_{0}$, the requirement of effective convection ( $\beta$ small) is that $q$ should be large. Thus, where our method works well is just the region where convection is unimportant, which justifies our neglect of convection. Where, on the other hand, our method becomes poor, because $q$ is large, it is also suggested that convection can no longer be ignored.

\section{THE DIFFERENCE EXPRESSION FOR RADIATIVE TRANSPORT}

We have seen that the temperature undergoes a very abrupt increase in the neighborhood of $10^{4}$. In order to find suitable difference expressions for $F$, defined earlier by a relation equivalent to

$$
H(I)=2 F(I)\left[W\left(I-\frac{1}{2}\right)-W\left(I+\frac{1}{2}\right)\right],
$$


we have made approximate integrations of the static equation for the various regimes.

At the photosphere temperatures where $\kappa$ increases approximately as $\kappa \sim T^{12}$, the static heat flow equation

$$
H=\frac{4}{3}(\sigma / \kappa)(d W / d M),
$$

with

$$
\kappa=W^{3} / a
$$

has the solution

$$
H M=\text { const }-\frac{4}{3} \sigma\left(a / 2 W^{2}\right)
$$

and we can form the difference equation

$H\left(M_{2}-M_{1}\right)=\frac{2}{3} \sigma\left(W_{1} / W_{2} \kappa_{1}+W_{2} / W_{1 \kappa_{2}}\right)\left(W_{2}-W_{1}\right)$.

On the other hand, where $\kappa=b / W$, the equation integrates to

$$
H M=\frac{2}{3}(\sigma / b)\left(W^{2}+\text { const }\right),
$$

so

$$
H\left(M_{2}-M_{1}\right)=\frac{2}{3} \sigma\left(1 / \kappa_{2}+1 / \kappa_{1}\right)\left(W_{2}-W_{1}\right) .
$$

For other power laws, similar expressions can be found but the constant in front would be different. We approximate the above two difference equations by

$$
H\left(M_{2}-M_{1}\right)=\frac{4}{3} \sigma \frac{\left(W_{2} / \kappa_{2}+W_{1} / \kappa_{1}\right)}{W_{1}+W_{2}}\left(W_{2}-W_{1}\right),
$$

which approximates the above forms for a strongly varying opacity - even if $\kappa_{2}$ differs by an order of magnitude from $\kappa_{1}$ and is exact for a constant opacity. This leads to

$$
F(I)=\frac{\overline{3} \sigma\left[W\left(\iota+\frac{1}{2}\right) / \kappa\left(I+\frac{1}{2}\right)+W\left(I-\frac{1}{2}\right) / \kappa\left(I-\frac{1}{2}\right)\right]}{\left.\left[\Delta M\left(I+\frac{1}{2}\right)+\Delta M\left(I-\frac{1}{2}\right)\right]_{L} W\left(I T^{\frac{1}{2}}\right)+W\left(I-\frac{1}{2}\right)\right]} .
$$

This has permitted the use of an interval near $10^{4}$ such that neighboring values of $W$ differ by more than an order of magnitude and yet the equations still make some sense. It is not claimed that this is a highly precise form of the difference equation, but it is a reasonable compromise between the varying requirements.

It appears from this, and experience with different expressions, that the essential requirement for a correct treatment of the opacity in a zone where the opacity in fact changes by a large factor is to use some approximation where the lowest opacity in the zone dominates the mean, rather than the greatest. The reason is that in the region of large opacity, the temperature gradient concentrates to a very large value and thereby largely nullifies the high opacity, leaving the heat flow to be determined in large measure by the lower opacity region.

\section{STATIC MODELS AND INITIAL CONDITIONS}

Following the discussion of the mass division, we are now able to integrate static models. These are integrated inward from the outside to some inner radius and some maximum temperature. The minimum radius is usually around $\frac{1}{5} R_{0}$ and the maximum temperature is usually greater than $10^{\circ}{ }^{\circ}$. In general, an envelope calculated in this way would not be acceptable if integrated to the center. However, the envelope properties in this region are not sensitive to the possibility of integrating in to the center, and apart from the values of $M$ and $L_{0}$, we are not here concerned with the central region.

In addition to the mass ratio of successive mass points which determines the fineness of the division, we must choose the initial $\Delta M$ of the first layer. In general, it was attempted to choose this to correspond to considerably less than unit optical depth, and normally it corresponded to optical depth less than 0.1. The reason for the fine zoning in the atmosphere was so that the velocity could be studied as a function of depth in the photosphere in order to see how the velocity observed in the Doppler shift of the spectral lines (assumed to correspond to optical depth 0.1 to 0.2 ) differed from the velocity of the photosphere itself. Some deviation from constancy of $\alpha$ was often made in the photosphere in order to satisfy the above requirement, and also at high temperatures (above $10^{5}{ }^{\circ}$ ) in order to avoid a rapid increase in $l$ near the center.

Since the treatment of heat transport was not accurate except in the average for the region near $10^{4}{ }^{\circ}$ and this average required a relatively large amplitude, calculations were usually initiated with a relatively large amplitude (about $\frac{1}{2}$ the final amplitude) motion by choosing a suitable deviation from the static model. It was desirable to choose this deviation as near as possible to a single normal mode so that the resulting motion would resemble as closely as possible a periodic oscillation. In general, of course, the initial conditions generated a superposition of several normal modes.

At this point, it may be appropriate to define a normal mode in a nonlinear problem of this kind. The definition probably must depend on the system. Our definition is based on a system where certain modes of motion are almost periodic and have almost 
constant amplitude. Where the motion can be followed for several periods at nearly fixed amplitude, we can define the fundamental as that periodic motion of longest period. The first harmonic is the periodic motion of the next shortest period, etc. In fact, the distribution of $U(r)$ closely resembles the linear case and the fundamental has no node in the body of the star, the first harmonic has one node, etc.

The initiation was chosen by superimposing on the static model some suitably chosen velocity distribution $U(M)$. It might be thought that in the function space $U(M)$, any mode could be generated completely free of contamination of other modes. It is true that suitably chosen distributions $U(M)$ can simulate arbitrary modes. However, these modes have a well-defined relative phase. It turns out that it is not possible to remove all the higher harmonics by choosing some particular $U(M)$. In general, it would be necessary to add a new correction with some new $U^{\prime}(M)$ at a time $90^{\circ}$ later in phase in order to eliminate unwanted modes. This added complication was not followed so that, in general, the modes studied had more or less contamination. This contamination was most serious in the mixture of fundamental and first harmonic. However, it was found that by averaging the results over several periods, this difficulty could be minimized.

The specification of the initial conditions was to superimpose on the static solution a velocity distribution which was actually $U(R)$ where $R$ is the radius of the mass point in question. For generating the fundamental, a power law $U \sim R^{n}$ where $n$ is near 6 is satisfactory. $n$ can be varied to minimize the unwanted higher harmonics. For generating the first harmonic, a distribution $U \sim a_{1} R^{n_{1}}+a_{2} R^{n_{2}}$ was used. Typical values would be $U_{1}=-20\left(R / R_{0}\right)^{10}+$ $6\left(R / R_{0}\right)^{5}$. The same form could also be used for the fundamental, i.e., $U_{0}=-10\left(R / R_{0}\right)^{10}-5\left(R / R_{0}\right)^{5}$. Harmonics above the first have not been initiated as yet but it is clear that a three-term velocity expression to give two nodes would be necessary for the second harmonic.

These expressions for $U(R)$ also demonstrate the requirements on the minimum radius. At the minimum radius $R_{1}$, the amplitude of these modes is very small provided the minimum radius is less than about $\frac{1}{3} R_{0}$. We have always chosen $R_{1}<\frac{1}{4} R_{0}$.

Although, as has been explained, the calculations were most reliable at fairly large amplitude, it was not at all easy to explore the maximum amplitude where the amplitude remains constant, by this method. The reason apparently has to do with a distortion of the normal mode when dissipation at large amplitude becomes important. This dissipation is large just in the surface zones where the shock waves develop and introduces changes in the phase relations. The result is that if the motion is initiated at large amplitude, the introduction of unwanted contamination by the first harmonic becomes more and more prominent and the resulting calculated motion deviates more and more from a simple periodic one. If the conditions are such that the first harmonic is strongly damped, then the large amplitude can be explored. However, in most cases of interest, the first harmonic is weakly damped if at all so that the very large amplitude initiation leads to considerable uncertainty in the results.

\section{RESULTS}

In order to illustrate the methods, the results obtained in the study of the following model will be discussed. The model has the defining parameters $M=0.75 \times 10^{33} \mathrm{~g}(\approx 0.4 M \odot), L=1.5 \times 10^{35}$ $\mathrm{erg} / \mathrm{sec}\left(M_{\mathrm{bol}} \approx+0.75\right), T_{\mathrm{e}}=6500^{\circ} \mathrm{K}$, and $R_{0}=$ $3.41 \times 10^{11} \mathrm{~cm}$. This is a possible model for an RR Lyrae (or cluster) variable star but the best models appear to have a mass nearer $M \odot$ and about twice the luminosity. The composition was chosen to be $X=$ mass fraction of $\mathrm{H}=0.548, Y=$ mass fraction of $\mathrm{He}=0.45$, and $Z=$ mass fraction of other elements $=0.002$.

The reason for choosing this example for illustration was that because of the relatively low mass, the envelope was of lower than normal density. This resulted in a considerably more rapid approach to the ultimate limiting amplitude than would be true for a more massive model. It was thus possible to follow this example to its limiting amplitude with a not unreasonable computing time.

Figures 1, 2, 3, 4 and Table I show various features

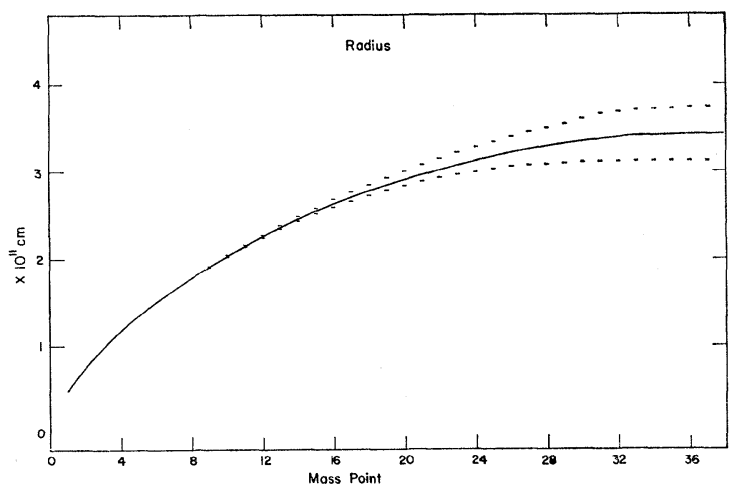

FIG. 1. The radii (cm) of the 38 mass points of the static model. The amplitude of the resulting oscillation of each point is shown by the limits. 
of the static model which preceded the dynamic calculation. The static model was set up with 38 mass zones and a surface mass zone of $3 \times 10^{24} \mathrm{~g}$

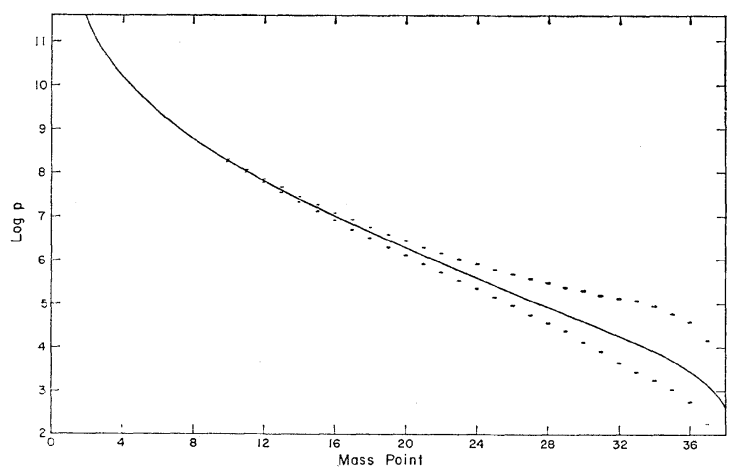

FIG. 2. The $\log _{10}$ of the pressure (dynes $/ \mathrm{cm}^{2}$ ) of the mass points of the static model. The amplitude of the oscillation is shown by the limits.

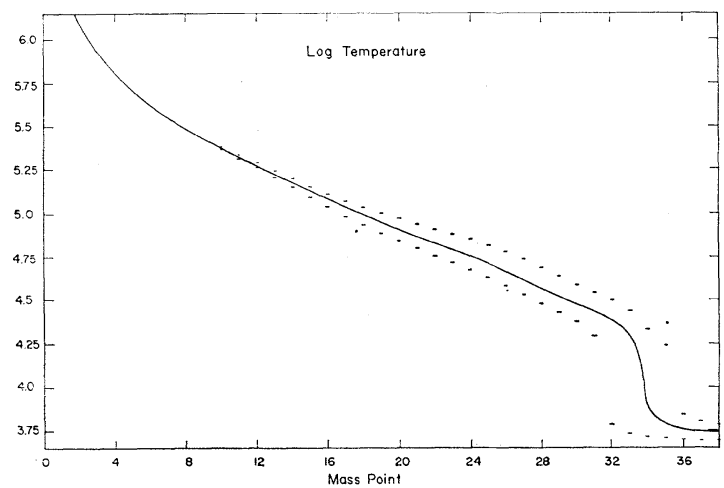

Fig. 3. The $\log _{10}$ of the temperature $\left({ }^{\circ} \mathrm{K}\right)^{\circ}$ of the mass points of the static model. The amplitude of the oscillation is shown by the limits.

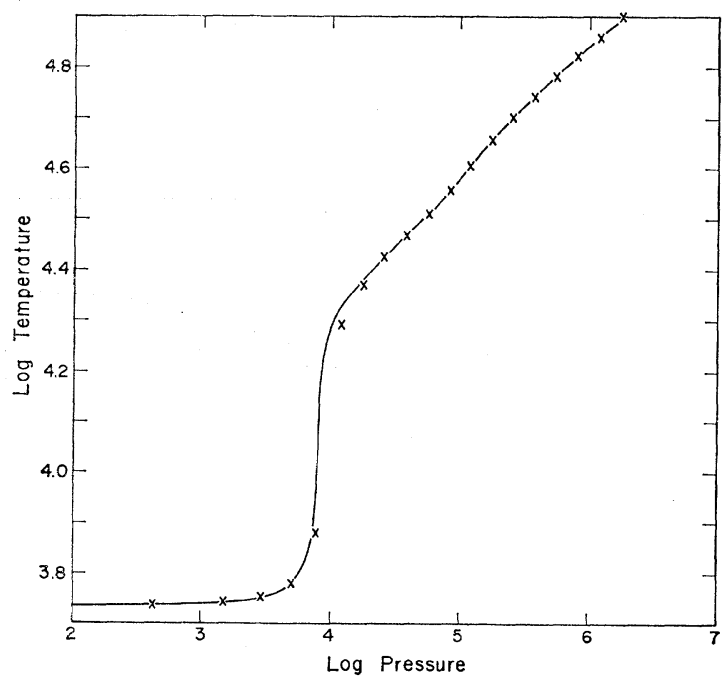

FIG. 4. The solid curve is the $\log _{10} T$ vs $\log _{10} P$ for a fine mass division. The points $X$ are the results of the 38 mass point static model. $\left(2.03 \mathrm{~g} / \mathrm{cm}^{2}\right)$; subsequent mass zones increased by a factor of 1.4 each step until at a temperature of about $2 \times 10^{5}{ }^{\circ} \mathrm{K}$ the ratio was made slowly to increase in order to keep the sound travel time from zone to zone nearly constant. This resulted in a ratio of 2.3 at the deepest mass zone which extended from $R=7.48 \times 10^{10} \mathrm{~cm}$ to $4.79 \times 10^{10} \mathrm{~cm}$. The (Rosseland mean) optical depths of the first few mass zones in the stellar photosphere are $\tau=0.008$ at the center of mass point 38, $\tau=0.034$ at $37, \tau=0.103$

TABLe I. The static model.

\begin{tabular}{lccccc}
\hline \hline $\begin{array}{l}\text { Mass } \\
\text { point }\end{array}$ & $\begin{array}{c}\text { Radius } \\
\Delta M\left(10^{24} \mathrm{~g}\right) \\
\left(10^{11} \mathrm{~cm}\right)\end{array}$ & $\begin{array}{c}\text { Temper- } \\
\text { ature } \\
\left({ }^{\circ} \mathrm{K}\right)\end{array}$ & $\begin{array}{c}\text { Pressure } \\
\left(\mathrm{dyn} / \mathrm{cm}^{2}\right)\end{array}$ & $\begin{array}{c}\text { Specific } \\
\text { volume } \\
\left(\mathrm{cm}^{3} / \mathrm{g}\right)\end{array}$ \\
\hline 38 & 3.00 & 3.43 & $5.46 \times 10^{3}$ & $4.29 \times 10^{2}$ & $6.94 \times 10^{8}$ \\
35 & 8.23 & 3.41 & $5.99 \times 10^{3}$ & $5.03 \times 10^{3}$ & $6.49 \times 10^{7}$ \\
32 & 22.6 & 3.38 & $2.37 \times 10^{4}$ & $1.80 \times 10^{4}$ & $1.44 \times 10^{8}$ \\
29 & 62.0 & 3.31 & $3.26 \times 10^{4}$ & $5.63 \times 10^{4}$ & $6.32 \times 10^{7}$ \\
26 & 170 & 3.21 & $4.55 \times 10^{4}$ & $1.74 \times 10^{5}$ & $3.00 \times 10^{7}$ \\
23 & 467 & 3.08 & $6.10 \times 10^{4}$ & $5.49 \times 10^{5}$ & $1.31 \times 10^{7}$ \\
20 & $1.28 \times 10^{3}$ & 2.91 & $7.95 \times 10^{4}$ & $1.82 \times 10^{6}$ & $5.17 \times 10^{6}$ \\
17 & $3.51 \times 10^{3}$ & 2.71 & $1.06 \times 10^{5}$ & $6.40 \times 10^{6}$ & $1.96 \times 10^{6}$ \\
14 & $9.64 \times 10^{3}$ & 2.46 & $1.49 \times 10^{5}$ & $2.44 \times 10^{7}$ & $7.24 \times 10^{5}$ \\
11 & $26.8 \times 10^{3}$ & 2.16 & $2.12 \times 10^{5}$ & $1.06 \times 10^{8}$ & $2.38 \times 10^{5}$ \\
8 & $83.9 \times 10^{3}$ & 1.79 & $3.05 \times 10^{5}$ & $5.89 \times 10^{8}$ & $6.12 \times 10^{4}$ \\
5 & $340 \times 10^{3}$ & 1.34 & $5.02 \times 10^{5}$ & $5.76 \times 10^{9}$ & $1.03 \times 10^{4}$ \\
2 & $2.72 \times 10^{6}$ & 0.75 & $1.22 \times 10^{6}$ & $3.03 \times 10^{11}$ & $4.76 \times 10^{2}$ \\
\hline \hline
\end{tabular}

at 36, $\tau=0.297$ at 35 , and $\tau=1.81$ at 34 . On Figs. 1,2 , and 3 , which show the run of $R, T, P$ against mass point, the amplitude of the resulting oscillation is also shown. The most noteworthy feature is the vanishingly small amplitude at small radii. It is this feature that justifies the neglect of the interior and replacing it by a boundary condition. A particular feature of the temperature distribution is shown in Fig. 3 plotted against mass point, and in Fig. 4 plotted against pressure. Starting from the surface at $T_{e} / 2^{2}$, the temperature rises at an ever increasing rate until the rise becomes almost vertical at $T \sim 10^{4}$ ${ }^{\circ} \mathrm{K}$. This takes place at a characteristic pressure $P_{1}$ which depends on $g$ and on the effective temperature approximately as $g^{\frac{2}{3}} T_{e}^{8}$. After the abrupt rise in temperature, the temperature soon approaches a law $T^{4} \propto P$ throughout the rest of the envelope. The solid curve in Fig. 4 shows $T(P)$ for a calculation with a very fine mass division in order to cover correctly the abrupt rise. The points marked $\times$ are those resulting from the 38 mass point division of the envelope. The close approximation of the points to the curve, even in the region of $10^{4}{ }^{\circ} \mathrm{K}$, shows that the difference approximations in the expression for the opacity of a mass point were successful.

The dynamical calculation which was initiated 
from this static model is illustrated in Figs. 5 and 6. They show the time-dependence of the velocity of mass point 36 (which approximates the level of for-

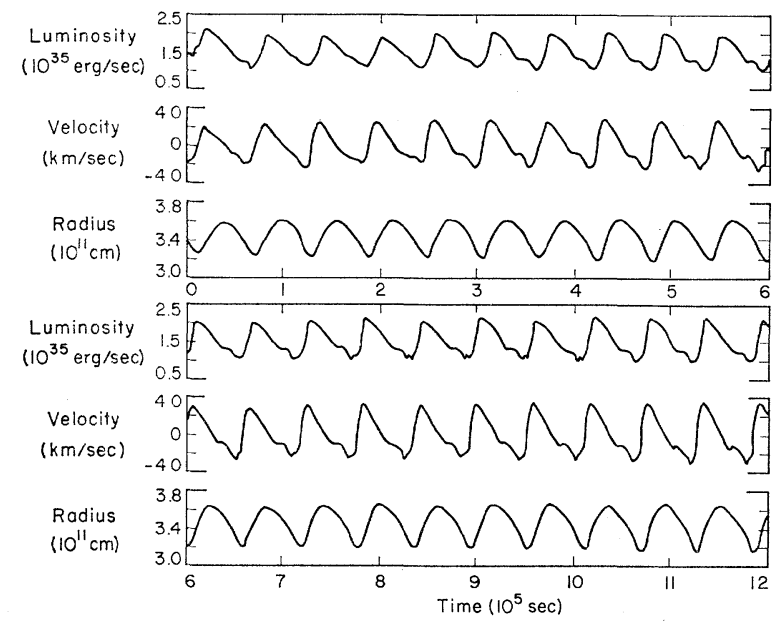

FIG. 5. The luminosity (ergs/sec), velocity $(\mathrm{km} / \mathrm{sec})$ of mass point 36 , and radius $(\mathrm{cm})$ of mass point 35 as a function of time for the first 20 periods of the motion.

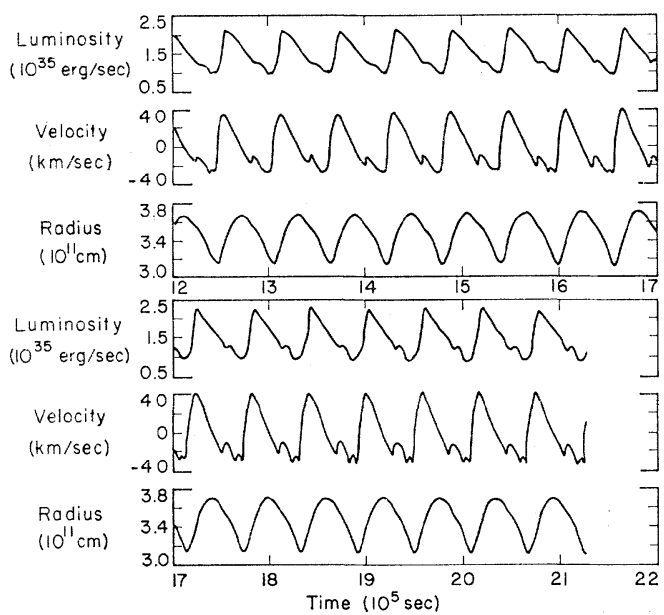

Frg. 6. The luminosity (ergs/sec), velocity $(\mathrm{km} / \mathrm{sec})$ of mass point 36 , and radius (cm) of mass point 35 as a function of time for the next 17 periods.

mation of weaker spectral lines), the radius of mass point 35 (which approximates the photosphere), and the luminosity. The initial conditions were $U=$ $-13.0\left(R / R_{0}\right)^{10}-7.0\left(R / R_{0}\right)^{5}(\mathrm{~km} / \mathrm{sec})$. It is apparent that the initial behavior deviates from a strict periodicity in that the fine detail of the velocity and light curves are variable, but in the main the curves repeat. This is ascribed to a mixture of modes or harmonics, in particular there is a small admixture of the first harmonic so that the second, fifth, etc., outgoing velocity maxima are larger than average. As time goes on, however, the higher harmonic content dies out and the curves approximate ever more closely to the periodic ones to be found toward the end of Fig. 6. The general features of these curves are shown in more detail in Fig. 7 where the velocity

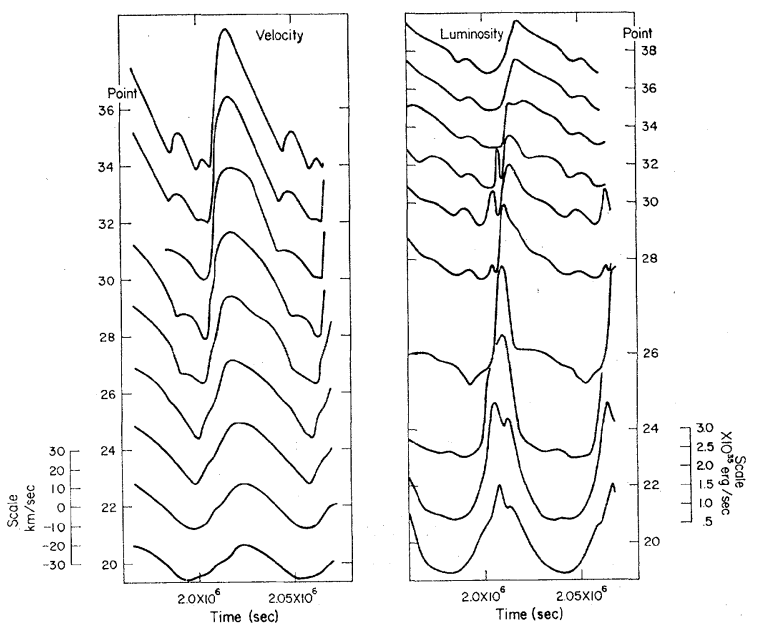

Fig. 7. The velocity for one period at the end of the calculation for each of mass points $36,34,32,30,28,26,24,22,20$. The luminosity for one period at the end of the calculation for each of mass points $38,36,34,32,30,28,26,24,22,20$.

for one period is shown for various mass points down to mass point 20 and also the luminosity at various levels is shown.

The ultimate velocity curve can be described as an outgoing shock which rapidly raises the velocity from about $30 \mathrm{~km} / \mathrm{sec}$ falling to about $30 \mathrm{~km} / \mathrm{sec}$ rising, followed by a slow rise to $45 \mathrm{~km} / \mathrm{sec}$ and then a decrease almost at constant acceleration to a point at minus $25 \mathrm{~km} / \mathrm{sec}$ where a second small shock or bounce raises the velocity by about $15 \mathrm{~km} / \mathrm{sec}$ after which it falls again to minus $30-35 \mathrm{~km} / \mathrm{sec}$ with another small bounce, after which the cycle repeats. The first "bounce" of the atmosphere can be followed in depth to the zone at mass point 27 . It will be interesting to be able to find in more detail the causes of this behavior.

The luminosity curve at the surface shows a sharp rise by a factor of about 2.5 which is nearly coincident with the shock in the velocity curve. After the peak, it decays slowly until a second small bump appears coincident with the "bounce" in the velocity curve. It can be ascribed to the heating associated with the compression accompanying the bounce. At greater depth-near zones 30 and 32-there is a small spike in the luminosity ahead of the main pulse. 
This seems to be due to a shock wave running ahead and can also be seen in the velocity curve. At still greater depth, near zones 26,24 , and 22 , the luminosity shows a very great peak of short duration. This peak arises from the helium ionization zone. The heat absorption in this zone delays the rise in temperature which causes an abnormally large temperature gradient and heat flux into the region. On expansion, the opposite effect prevails until the flux is reduced to a small value again to await the next cycle. The phase delay and general form of the luminosity curve is first apparent at mass point 28 just above the helium zone. The delay becomes progressively larger nearer the surface and the luminosity develops its final form on passage through the hydrogen zone.

With the choice of conditions as outlined, the motion was followed until the amplitude had almost ceased to grow. Experience has shown that the most reliable measure of the amplitude - that measure that is most independent of the harmonic mixture- is the gain in peak kinetic energy of the envelope in a period. Actually, to minimize the influence of the first harmonic which has a period about $\frac{3}{4}$. of the fundamental, and therefore gives a super period of about 3 times the fundamental, the increase in kinetic energy was measured for 3 periods. Figure 8 shows the mean fractional increase $\Delta \mathcal{E} / \mathcal{E}$ for the kinetic energy per period plotted against the peak kinetic energy. It is apparent that the curve can be extrapolated to a final peak energy not much greater than the maximum already present. In this way, the approach to the maximum amplitude was followed. The curve can be crudely fitted by $\Delta \varepsilon /$ period $=$ $a \varepsilon\left(1-\varepsilon / \varepsilon_{\max }\right)^{2}$, where $a=0.04$.

Attempts have been made to find measures by which the calculated pulsations can be compared to observed ones. One measure is the phase relation between the luminosity and the dynamics. The simplest measure of this phase relation is to be found in the

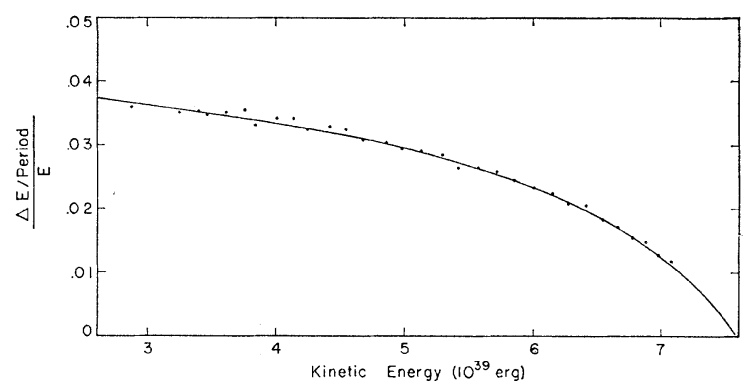

Fig. 8. The fractional increase per period of the peak kinetic energy of the envelope as a function of that peak energy.

phase relation between the luminosity and velocity curves since these curves are so similar. However, since the curves are nonsinusoidal, this phase difference must be defined with respect to some characteristic feature of the observations and calculations. Although, observationally, it is common to use the peak luminosity for this purpose, it does not seem that that is the most convenient for comparison with theory since the peak may be sensitive to small details in shape. We have chosen to use the time when $L=L_{0}$ the mean luminosity (on the rising branch) as a time point in the luminosity curve. Similarly, we have chosen the time when the velocity $=0$ (at minimum radius) as a suitable epoch on the velocity curve. We then define a phase lag as the lag of the luminosity curve behind the velocity curve divided by the period of the motion. Clearly, in this notation

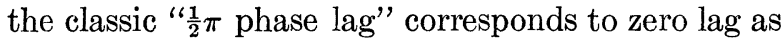
defined here. The phase lag is shown in Table II as a function of time for the calculation which was dis-

TABLE II. The dynamical model.

\begin{tabular}{|c|c|c|c|c|c|c|c|c|c|}
\hline $\begin{array}{l}\text { Number of } \\
\text { periods } \\
\text { after start }\end{array}$ & $\frac{\Delta R}{R}$ & $\frac{I L A}{L_{0} \times \text { period }}$ & $\begin{array}{c}\text { Period } \\
\left(10^{3} \mathrm{sec}\right)\end{array}$ & $\begin{array}{c}\text { Phase } \\
\text { lag }\end{array}$ & $R$ & $\begin{array}{c}\mathcal{E} \\
\left(10^{39} \mathrm{erg}\right)\end{array}$ & $\frac{\Delta \varepsilon}{\mathcal{E}}$ & $\begin{array}{c}U_{\max } \\
(\mathrm{km} / \mathrm{sec})\end{array}$ & $\begin{array}{c}-U_{\min } \\
(\mathrm{km} / \mathrm{sec})\end{array}$ \\
\hline 2 & 0.1082 & 0.0831 & 58.63 & 0.127 & 1.302 & 2.874 & 0.036 & 24.36 & 20.11 \\
\hline$\overline{5}$ & 0.1154 & 0.0919 & 58.70 & 0.105 & 1.256 & 3.244 & 0.035 & 26.92 & 23.80 \\
\hline 8 & 0.1216 & 0.0961 & 58.90 & 0.100 & 1.265 & 3.603 & 0.035 & 28.92 & 24.39 \\
\hline 11 & 0.1281 & 0.0997 & 58.77 & 0.096 & 1.285 & 4.003 & 0.034 & 30.57 & 26.30 \\
\hline 14 & 0.1350 & 0.1038 & 58.70 & 0.091 & 1.301 & 4.414 & 0.033 & 32.26 & 27.93 \\
\hline 17 & 0.1438 & 0.1067 & 58.66 & 0.089 & 1. 348 & 4.874 & 0.031 & 34.05 & 28.65 \\
\hline 20 & 0.1499 & 0.1103 & 58.68 & 0.086 & 1. 359 & 5.286 & 0.028 & 35.77 & 28.25 \\
\hline 23 & 0.1568 & 0.1130 & 58.69 & 0.085 & 1.388 & 5.724 & 0.026 & 37.57 & 27.50 \\
\hline 26 & 0.1633 & 0.1152 & 58.70 & 0.087 & 1.418 & 6.149 & 0.022 & 39.46 & 28.04 \\
\hline 29 & 0.1690 & 0.1187 & 58.72 & 0.085 & 1.424 & 6.540 & 0.019 & 41.31 & 30.34 \\
\hline 32 & 0.1726 & 0.1213 & 58.76 & 0.080 & 1.423 & 6.879 & 0.014 & 42.44 & 31.73 \\
\hline 35 & 0.1751 & 0.1234 & 58.76 & 0.075 & 1.420 & 7.144 & 0.011 & 43.59 & 32.22 \\
\hline 38 & 0.1775 & 0.1256 & 58.77 & 0.071 & 1.413 & 7.365 & 0.009 & 44.56 & 32.70 \\
\hline 41 & 0.1794 & 0.1272 & 58.77 & 0.069 & 1.410 & 7.542 & 0.007 & 45.30 & 33.41 \\
\hline
\end{tabular}


cussed above. We see that the lag was slightly positive at small amplitude and decreased somewhat as the amplitude approached its maximum value. The correspondence with phase lags as observed is, however, good.

Another measure of the nature of the motion is to be found in the ratio of luminosity amplitude to the radius amplitude. To this end, we define the radius amplitude as $\left(R_{\max }-R_{\min }\right) / R_{0}=\Delta R / R_{0}$. As in other measures, if the motion involves a superposition of harmonics, we can considerably reduce the error by averaging over 3 periods since this removes the principal source of variation due to the first harmonic because its period is $\approx \frac{3}{4}$ the period of the fundamental. In the technique employed here for treating the radiation transfer, the temperature and luminosity tend to oscillate around their true values because of the finite size of the mass zones near $10^{4} \circ$. As a result of this, the luminosity amplitude is not accurately given and we have found that the $\int\left(L-L_{0}\right) d t$ is a better quantity to use. We then take the amplitude of variation of the above quantity which is

$\int_{L=L_{0} \text { rising }}^{L=L_{\mathrm{o}} \text { falling }}\left(L-L_{0}\right) d t=\mathrm{ILA}$

(the integrated luminosity amplitude).

Then we define a measure of the luminosity amplitude as ILA $/ L_{0} \times$ period. We are now able to define a ratio $R=(\Delta R / R) /\left(\mathrm{ILA} / L_{0} \times\right.$ period $)$. $R$, as well as $\Delta R / R_{0}$ and ILA $/ L_{0} \times$ period, is shown in Table II for various times during the calculation.

It is apparent that $R$ is fairly constant, independent of amplitude. In another paper we will find that $R$ is a very useful measure of the pulsation. It is particularly so since it is reasonably independent of amplitude and can therefore be studied in the intermediate amplitude situation which is computationally easier to examine than the full amplitude problem being discussed here.

In order to explore the basic physics of the pulsation, we have plotted in Fig. 9 the $P-V$ diagrams for a number of the mass points over one period of the motion at $t=18$ periods from the start. By integrating the area inside these loops, we can find where the energy is being generated and where dissipated in the model. The results of this calculation are shown in Fig. 10. It is apparent that there are two zones of energy generation, one near mass points 26 and 27 and one near mass points 32 and 33. By reference to the static model in Table I, we see that the temperature in the zone 27 is $4.0 \times 10^{4}$, and in zone 26 is

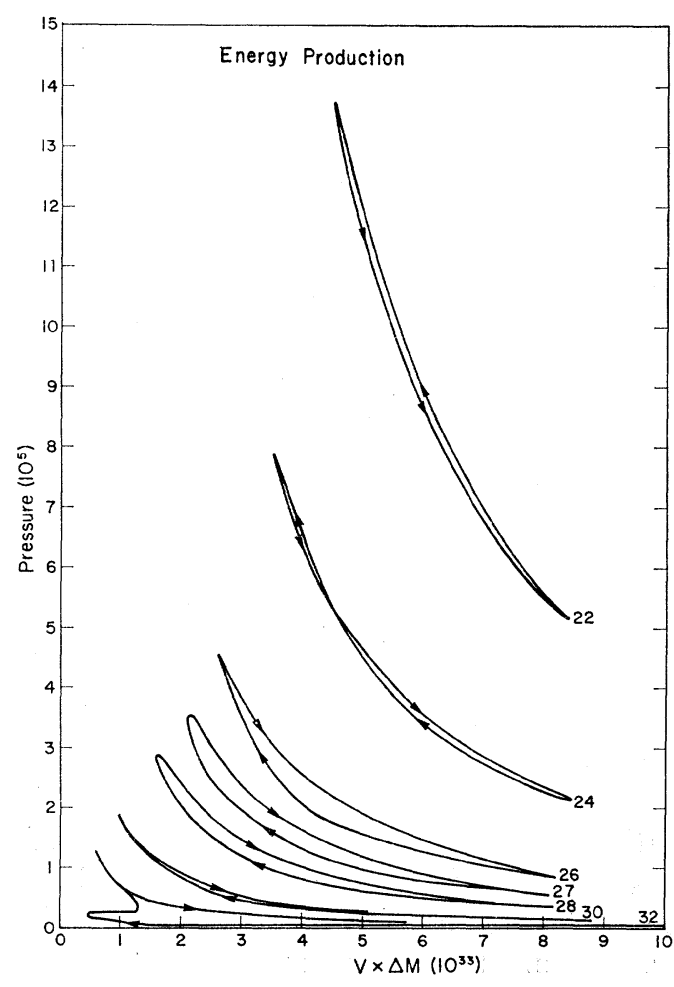

FIG. 9. $P-V$ diagrams for mass points $32,30,28,27,26$, 24 , and 22 after 18 periods. The abscissa is the total volume for each mass point.

$4.5 \times 10^{4}$, and corresponds to the second ionization of He which takes place just at these conditions. The temperature at zone 33 is $2.0 \times 10^{4}{ }^{\circ}$ and at 32 is $2.4 \times 10^{4}$. This temperature is clearly related to the ionization of hydrogen and the first ionization of $\mathrm{He}$ and it is apparent from Fig. 3 that these points cycle through the ionization conditions of hydrogen and helium. The total of the positive contributions

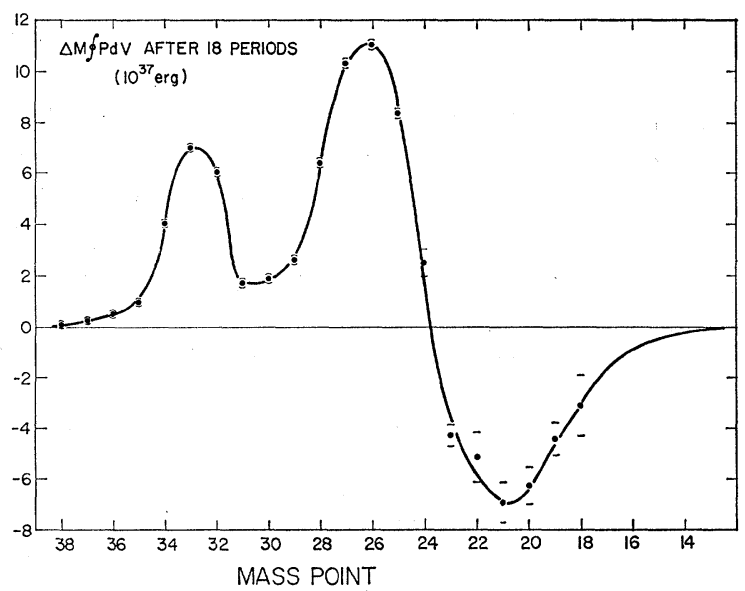

Fig. 10. The work done per period (positive or negative) for various mass points after 18 periods. 
to the energy production is $6.3 \times 10^{38}$ which is $7.2 \%$ of $L_{0} \times$ period (at maximum amplitude, about $11 \%$ of the luminous flux is converted into mechanical energy per period). Of this, the hydrogen ionization zone produces $33 \%$ and the second helium ionization produces $67 \%$. The total dissipation at this time is $4.9 \times 10^{38} \mathrm{erg} /$ period and the increase in kinetic energy per period is $1.5 \times 10^{38} \mathrm{erg} /$ period. The reason for the sensitivity of this quantity to slight errors in the treatment of the hydrogen zone is apparent.

The importance of the second He zone has been demonstrated by Zhevakin ${ }^{5}$ and developed more fully by $\mathrm{Cox}^{7}$ and by Baker and Kippenhahn. ${ }^{6}$ The significance of the hydrogen zone was suggested by Eddington ${ }^{15}$ who, however, was unable to correctly develop its consequences. The hydrogen zone was discussed rather more fully by the author ${ }^{4}$ where the possibility that it was an important contributor to the instability was pointed out. ${ }^{16}$

The basic reason for the energy generation in the ionization zones has already been discussed in I. We may say, simply, that because of their small $\gamma$ (ratio of specific heats) and high heat capacity, the ionization zones find themselves cooler, relative to their surroundings, on adiabatic compression and hotter on adiabatic expansion. This means that they will absorb heat when compressed and give it off when expanded. This behavior is just the behavior appropriate for the generation of work and is just the behavior we see exemplified in the ionization zones in Figs. 9 and 10.

The importance of the ionization zones as mentioned above, is also conditioned by their depth in the envelope. If $T_{e}$ is too great, the ionization zones are too near the surface and consequently will involve too little material to provide any significant heat capacity. As a result, the phase delay of the temperature is too small and the energy production is reduced so that the dissipation will dominate and the star will be stable. The boundary to the unstable region on the low $T_{e}$ side is more obscure. Estimates suggest it may be associated with the onset of effective convection. Since convection has been ignored in these calculations, we are unable to settle this question at this time.

In regions of constant ratio of specific heats, it is

${ }^{15}$ A. S. Eddington, Monthly Notices Roy. Astron. Soc. $101,182(1941)$.

${ }_{16}$ In that paper, the contribution of the hydrogen zone to the pulsation was considerably overestimated because of an incorrect surmise about the value of $\Delta V$, the change in volume. It was guessed that the whole expansion of the star might arise from the expansion of the hydrogen zone. In fact, only about $19 \%$ arises there, about $37 \%$ in the $\mathrm{He}$ zone and the rest deeper in the envelope. also known that the dependence of opacity on temperature and density is very important. The usual dependence $\kappa \sim 1 / V T^{3.5}$ leads to dissipation since the heat transport is greatest when the material is compressed adiabatically. This accounts for the dissipation in the deeper regions of the envelope.

The time-dependent calculation that has just been discussed depends on many somewhat arbitrary parameters in addition to those that determine the static solution. In most cases, the choices made were a compromise between accuracy and speed. In order to evaluate these compromises, a series of test calculations was made to explore the sensitivity of the results to the choices that were made. These test runs were carried in time only far enough to evaluate the trend of the results. This was about 6 periods of the motion. The test runs were then compared with the corresponding time portion of the main calculation. The quantities that were insepected in this comparison were just those quantities listed in Table II which were chosen as the most suitable simple numerical measures of the motion.

Before comparing the test runs with the main calculation, it is worth inspecting Table II in order to understand the sensitivity of the results. Some quantities in the table behave very smoothly and others somewhat irregularly. There are two sources of the irregularity. The first has to do with the initial conditions which have introduced various high harmonics. The averaging over three periods that has been carried out removes some, but not all, of this irregularity. A more serious irregularity results from the coarse zoning near $10^{4}{ }^{\circ}$. Although the calculation is able to deal with the principal effects of the opacity behavior in this region, the coarse zoning leads to residual irregularities in the temperature and luminosity and energy production. In particular, these quantities are still somewhat sensitive to the location of the zone boundary in the $10^{4}{ }^{\circ}$ region at the minimum luminosity of the cycle. Depending on where this zone boundary is, there is either a larger or smaller than normal value of ILA and also of $\Delta \varepsilon$. Since, as the amplitude grows to the maximum, the location of this temperature at minimum crosses about one zone, there is a corresponding variation of ILA and of $\Delta \varepsilon$ to be found. In particular, we note that at the initiation time $\Delta \varepsilon$ is near its maximum positive excursion and ILA its minimum. As a result, we find these quantities unusually sensitive to any changes-these almost certainly leading to a decrease of $\Delta \mathcal{E}$ and an increase of ILA.

With this in mind, we have examined the test runs for significant changes. The series that was carried 
out is displayed in Table III. The parameters were explored and the results were as follows. In this discussion, only noticeable or significant effects are mentioned. Quantities not mentioned did not change appreciably.

The effect of the precision of the iteration procedure for the temperature equation was explored in the first test. This precision is defined by the maximum value of $\Delta T(I)$ that is permitted without iterating again. Normally, this has been $1^{\circ} \mathrm{K}$ and the location where the error is usually largest is near $10^{4}$. An average 3 to 4 iterations is usually needed for this precision. A value of $\Delta T=10^{\circ} \mathrm{K}$ was tried in test 1 , the only perceptible change was a reduction of $\Delta \mathcal{E} / \mathcal{E}$ by $1 \%$ of its value. This was fortunate since the latter half of the main calculation had been carried out with this value in order to improve the rate of convergence which had become worse because of the strong shocks. Also, many of the subsequent tests used this same value of $10^{\circ} \mathrm{K}$.

The second test explored an over-all reduction of the time between cycles by $1 / \sqrt{2}$ by reducing $l_{0}^{2}$ (the maximum permitted value of $c^{2} \Delta t^{2} / \Delta R^{2}$ ) by two. The result was again that no significant changes were found. The most sensitive quantity $\Delta \varepsilon / \mathcal{E}$ was reduced by $1 \%$. This result was in marked contrast with the methods used in $I$ which are also discussed in Appendix B.

Test 3 involved an increase of $C_{Q}$ from 1 to 4 . This change led to a reduction of $\Delta \mathcal{E} / \mathcal{E}$ by $38 \%$. This led to a reduction in amplitude but no noticeable change in the amplitude insensitive quantities such as the ratio $R$, the period and the phase lag. Test 4 then reduced $C_{Q}$ to 0.2 and used $\Delta T=10^{\circ}$ since this small a value of $C_{Q}$ leads to noticeable fluctuation of the points. Compared to the standard, this changed only $\Delta \varepsilon / \mathcal{E}$ which was increased by $10 \%$ of its value. The results of these tests show that the choice of $C_{Q}=1$ for the parameter in the artificial viscosity was satisfactory. It is also worth noting that the treatment is marginal in this respect since $C_{Q}$ can not be made much smaller without an undesirable increase in fluctuation.

Test 5 explored the effect of increasing $\alpha$, the ratio of masses of successive zones, to 1.6 and a consequent reduction in the number of zones to 28 . This reduced the computing time for the same number of periods to $60 \%$ of its value. However, significant changes in results were found. The phase lag was reduced by 0.025 , the period was increased by $0.5 \%$, and the $\Delta \varepsilon / \varepsilon$ was reduced by $20 \%$. Test 6 reduced $\alpha$ to 1.2 , increasing the number of zones to 66 . The computing time was doubled. The principal effect was an increase of $\Delta \varepsilon / \mathcal{E}$ by about $6 \%$. In addition, the period was reduced by $0.5 \%$, $R$ was increased by $3 \%$, and the phase lag was reduced by 0.006 . The evidence suggests that the small effects other than $\Delta \varepsilon / \mathcal{E}$ are largely spurious.

In test 7 , the surface mass zone was reduced to $1.0 \times 10^{24} \mathrm{~g}$. No significant changes resulted except an almost $30 \%$ increase in computing time. The choice of the topmost zone sizes was in fact dictated by a desire to reproduce the dynamics of the region above the photosphere where the spectral lines are formed. Since the topmost zone shows deviations in its motion, this meant that the second or third zone from the top should still be at optical depth about 0.1 . The test indicates that the motion computed was apparently reliable.

Tests 8,9 , and 10 were attempts to explore the effects of the choice of the lower boundary $R_{1}$. In test 8 the same zoning was used but the two lowest zones were deleted, increasing $R_{1}$ to $9.72 \times 10^{10}$. There was a significant reduction of $\Delta \varepsilon / \varepsilon$ by $22 \%$. The phase lag was increased by $0.013, \Omega$ increased by $3 \%$, and the period reduced by $0.2 \%$. Tests 9 and 10 attempted to separate these effects into those due to the extra large zones at the bottom and those due to a change in $R_{1}$. Thus, test 9 maintained $\alpha$ constant to $R_{1}=5.25 \times 10^{10}$. There resulted a $6.5 \%$ reduc-

TABLE III. The test runs.

\begin{tabular}{|c|c|c|c|c|c|c|c|c|c|c|c|}
\hline \multirow[b]{2}{*}{ Parameter } & \multirow[b]{2}{*}{ Standard } & \multicolumn{10}{|c|}{ Test } \\
\hline & & 1 & 2 & 3 & 4 & 5 & 6 & 7 & 8 & 9 & 10 \\
\hline$\Delta T^{\prime}\left({ }^{\circ} \mathrm{K}\right.$, maximum error in iteration of temperature $)$ & 1 & 10 & 1 & 1 & 10 & 10 & 10 & 10 & 10 & 10 & 10 \\
\hline$l_{\max }$ (maximum value of Courant parameter $c \Delta t / \Delta R$ ) & 0.6 & 0.6 & 0.3 & 0.6 & 0.6 & 0.6 & 0.6 & 0.6 & 0.6 & 0.6 & 0.6 \\
\hline$C_{\boldsymbol{Q}}$ (artificial viscosity parameter) & 1 & 1 & 1 & 4 & 0.2 & 1 & 1 & 1 & 1 & 1 & 1 \\
\hline$\alpha$ (ratio of successive mass zones) & 1.4 & 1.4 & 1.4 & 1.4 & 1.4 & 1.6 & 1.2 & 1.4 & 1.4 & 1.4 & 1.4 \\
\hline$\Delta M_{0}\left(\right.$ surface mass zone in units of $10^{24} \mathrm{~g}$ ) & 3.0 & 3.0 & 3.0 & 3.0 & 3.0 & 3.0 & 3.0 & 1.0 & 3.0 & 3.0 & 3.0 \\
\hline$R_{1}$ (radius of inner boundary, $10^{10} \mathrm{~cm}$ ) & 4.8 & 4.8 & 4.8 & 4.8 & 4.8 & 6.3 & 3.4 & 1.8 & 9.7 & 5.2 & 7.6 \\
\hline Temperature above which $\alpha$ is allowed to increase $\left(10^{5}{ }^{\circ} \mathrm{K}\right)$ & 2.1 & 2.1 & 2.1 & 2.1 & 2.1 & 2.1 & 2.1 & 2.1 & 2.1 & 20 & 20 \\
\hline
\end{tabular}


tion in $\Delta \varepsilon / \varepsilon$ compared to the standard problem and no other significant changes. Test 10 then, with constant $\alpha$, increased $R_{1}$ to $7.65 \times 10^{10}$. There resulted a reduction of $\Delta \mathcal{E} / \mathcal{E}$ of $12 \%$ compared to the standard problem. In addition, $\Omega$ increased by $2 \%$, the period decreased by $0.1 \%$, and the phase lag increased by about 0.005 .

Following the above tests, two tests (11 and 12) were made to investigate the approach to the limiting amplitude from different initial conditions. Test 11 initiated a motion with initial kinetic energy equal to that present in the main calculation after 26 periods. There was, at first, considerable dissipation due to a shock running ahead of the main luminous flux peak and releasing energy at the surface in a flash which preceded the principal peak. This behavior continued until the kinetic energy was reduced by about $5 \%$ to equal that present in the main calculation at 24 periods. At that time, the results of test 11 showed every indication of joining those of the main calculation. This took about 6 periods. A test was then made to initiate the calculation at a kinetic energy equal to the (extrapolated) maximum in the main calculation. This led to a series of violent shocks (only every third period since the shock results from a strong first harmonic component) with emission of early flashes from the star. The kinetic energy in this way was diminished by about $25 \%$ after six periods and the test was discontinued. These trials show that great ingenuity in the initial conditions must be used to initiate these motions at near their maximum amplitude without introducing violent harmonic admixtures which are slow to decay. These tests also tend to confirm the independence of the final motion to the initial conditions which, of course, is to be expected on physical grounds.

As a result of these tests, it can be concluded that the technique used was reasonably reliable. It is also apparent that the result that is most sensitive is the magnitude of the instability $\Delta \varepsilon / \mathcal{E}$. This, of course, was expected since it is a derivative. For envelopes of significantly lower $T_{e}$, the surface opacity is greatly reduced and the surface zones can be taken much thicker. This permits a reduced value of $\alpha$ for a fixed number of zones. However, the other critical pointthe number of zones crossed by the level $10^{4}{ }^{\circ}$ in a period-is reduced and the results on $\Delta \mathcal{E} / \mathcal{E}$ tend to become, for this reason, more uncertain.

\section{CONCLUSION}

We have shown that it is possible to compute the pulsation behavior of a stellar envelope (when con- vection can be ignored). It is thereby possible to explore in detail all of the peculiarities shown in these motions as well as to explore the causes of the instability. The attempt to extract information about the stars from the wealth of observational material which is available, or can be obtained, can now be greatly extended. This program is now being pursued and results on RR Lyrae stars will soon be reported.

\section{ACKNOWLEDGMENT}

It is appropriate that this paper should be part of a salute to J. R. Oppenheimer. It was under his guidance that the author did his graduate work, and the inspiration of Oppenheimer was responsible not only for the thesis but also for subsequent work of the author. It was during a sabbatical year spent as a guest at the Institute for Advanced Study that the work on this problem was initiated and the author is particularly indebted to J. R. Oppenheimer for making that stay possible. Finally, it was at Los Alamos, under Oppenheimer's direction and encouragement, that the author first learned about hydrodynamics, and it was there that computational techniques for hydrodynamics were developed. It is particularly gratifying to note that techniques, developed for the design of atomic weapons, are here employed to study the dynamics of stars.

\section{APPENDIX A. SPECIAL TECHNIQUES}

A facet of the calculation where numerical difficulties arise is the treatment of the deeper regions of the envelope. These regions are characterized by very small changes in $T$ (or $W$ ) and in $R$ during the motion and yet the change in internal energy and in potential energy associated with these small changes are very large because of the very large mass per zone. As a consequence, round-off errors of $10^{-8}$ in $W$ or $R$, associated with the computer can lead to large errors in the energetics and in the behavior of the system. In the interest of speed, it was desired to avoid double precision calculation so this problem was handled by separately storing both the original value of the $W$ and of $R$ and also the accumulated small changes or increments in $W$ and $R$. The roundoff error in the increments was negligible and the present value of $W$ or $R$ could always be found by a single operation of adding the accumulated increments to the original value without having a succession of round off-errors.

The equation of state and opacity were used in the form of a table, stored in the computer, up to $T \approx 3.1 \times 10^{5}$. Above this temperature, both helium and hydrogen are essentially completely 
ionized and the material can be treated as a perfect gas. Also, the opacity can be approximated by a simple analytic law in this region. The treatment then involved using a table for all mass points initially below $T=2.4 \times 10^{5}{ }^{\circ}$ and using a simple formula for all points initially above $T=2.4 \times 10^{5}$. By assigning the same law at all times to each point and making a (slight) change in the equation of state and opacity at a given mass layer, the possibility of inadvertently supplying energy by changing equations of state in time was avoided. In addition, the storage requirement was reduced by the limited table.

\section{APPENDIX B. COMPARISON WITH THE NUMERICAL CALCULATION OF $I$}

In the first $\operatorname{attack}^{4}$ on the numerical calculation of pulsation, there were two objectives. The first was to demonstrate the existence of the pulsation instability associated with hydrogen ionization and the second was to demonstrate the feasibility of direct numerical integration of the dynamics of an envelope. Both these objectives were reached and the existence of exponentially growing pulsations was demonstrated numerically. Apart from these objectives, it was desired to keep the calculation as simple and fast as possible.

The treatment differed from the present one in several respects. The opacity law was crude, the equation of state was somewhat approximate, and the envelope was plane rather than spherical. In addition, there were two significant differences in the numerical work. First, the hydrodynamics was treated by an implicit rather than an explicit method. The implicit method is able to take longer time steps than permitted by the Courant condition but is unable to handle shocks. The other difference lay in the treatment of the nonlinear heat flow equation. It was approximated by expanding all functions of $T$ by Taylor series and so linearizing to get an explicit set of coupled equations for the $\Delta T$ 's. This procedure necessarily is less accurate than the one in use in the present treatment, which solves the set of coupled nonlinear equations by iteration.

It is not known which of the two numerical differences was responsible for the fact that there was in the calculation a numerical damping of pulsation which depended on the size of the time interval.

In order to get a reliable result for the exponential growth rate, it was necessary to recalculate for several values of $\Delta t$ and extrapolate to zero $\Delta t$. Because of this problem and because of the inability to handle shocks, the current treatment was introduced.

\section{APPENDIX C. THE PROBLEM OF VIBRATIONS OF A NONLINEAR SYSTEM}

Starting with the work of Fermi, Pasta, and Ulam on numerical integration of a loaded string with nonlinear coupling, mathematical interest in such nonlinear problems has increased. ${ }^{17}$ The author believes that the experience here in solving what amounts to $2 N$ nonlinear coupled difference equations, may be of interest in other cases.

The essential result here is that periodic solutions can be found. These are strictly periodic but of fixed amplitude only if we confine ourselves to the large amplitude case, but also there are almost periodic solutions of arbitrary amplitude. Here, the designation "almost periodic" refers to the slowly growing or decaying solutions. This demonsrration of periodic solutions is in some contrast to the results of Fermi et al., who found that there was no indication of equipartition but also no indication of periodic solutions, but rather that the energy migrated from the fundamental mode to higher modes and back again. The different results here probably are essentially related to the presence of dissipation so that when discontinuities appear, they are able to lead to dissipation rather than arbitrary higher modes. Our results in this problem remind one, in fact, of "limit cycle" type problems except that we here deal with partial differential equations rather than ordinary ones.

\footnotetext{
${ }^{17}$ For references, see E. A. Jackson, J. Math. Phys. 4, 686 17 For
$(1963)$.
} 